\title{
Discharge flow of a bidisperse granular media from a silo
}

\author{
M. Benyamine and M. Djermane \\ Laboratoire FIMAS, Université de Béchar, Boîte Postale 417, Béchar 08000, Algeria
}

B. Dalloz-Dubrujeaud and P. Aussillous*

Aix-Marseille Université, CNRS, IUSTI UMR 7343, 13013 Marseille, France

(Received 9 January 2014; revised manuscript received 20 June 2014; published 2 September 2014)

\begin{abstract}
The discharge flow in a cylindrical and a rectangular silo using both monodisperse and bidisperse mixtures of spherical glass beads is studied experimentally. The flow rate is measured using a precision balance for a large range of particle diameters, size ratios, and outlet diameters. A simple physical model is proposed to describe the flow of bidisperse mixtures. It gives an expression for the flow rate and predicts that the bulk velocity follows a simple mixture law. This model implies that a mixture diameter cannot be simply defined. Moreover it is shown that bidisperse granular media allow for the transport of coarse particles below their jamming conditions.
\end{abstract}

DOI: 10.1103/PhysRevE.90.032201

PACS number(s): 45.70.Mg, 81.05.Rm

\section{INTRODUCTION}

The discharge flow of granular mixtures from silos is of practical interest in many processes (for example, in food, mining, ceramic, paint, and pharmaceutical industries). The prediction of the discharge rate is of primary importance to assure reproducible and efficient handling in plant operations. Until now, most efforts have been made in the study of the discharge rate of monodisperse particles in silos. Nedderman et al. [1] published a review article in 1982 on the discharge rate through orifices. One of the simplest and most used empirical expressions for the flow rate was proposed by Beverloo et al. [2] for flat-bottomed silos,

$$
Q=C \rho \phi g^{1 / 2}(D-k d)^{5 / 2},
$$

where $Q$ is the mass flow rate, $g$ is the gravitational acceleration, $\rho$ is the particle density, $\phi$ is the particle volume fraction, $D$ is the outlet diameter, $d$ is the particle diameter, and $C$ and $k$ are fitted parameters. This model is based on dimensional analysis with the concept of a free fall arch at the outlet which was introduced first by Hagen in 1852 (traducted in [3]) and recently observed experimentally by Janda et al. [4]. This arch is supposed to scale with $D$ giving a velocity at the outlet of $v_{o} \approx \sqrt{g D}$ and a flow rate of $Q \approx \phi \rho v_{o} S_{o} \approx \phi \rho \sqrt{g D^{5}}$. This scaling does not account for the dependence of the flow rate on the particle diameter $d$, which has been introduced empirically by Hagen (traducted in [3]) or Beverloo et al. [2] considering a reduced outlet length $(D-k d)$, where $k$ is a fitting parameter explained lately by the concept of a useless zone (an "empty annulus") for the flow close to the outlet walls [5]. This empirical expression is widely used as it is in fairly good agreement with most experimental results. However, silos' discharge of monodisperse granular media remains an open subject of research as shown by the abundant literature available.

In practice not many systems are monosized. Concerning the discharge of a granular mixture through silos, most of the studies were devoted to the segregation phenomena, and few authors have looked into the factors influencing the

\footnotetext{
*pascale.aussillous@univ-amu.fr
}

mixture flow rate. Arteaga and Tüzün [6] studied the flow of a bidisperse mixture in a cylindrical and a conical silo. They described two behaviors corresponding to a coarse continuous phase and a fine continuous phase, depending on the fine mass fraction $\left(X_{f}\right)$ and the size ratio $\left(r=d_{c} / d_{f}\right)$, between the coarse particles $\left(d_{c}\right)$ and the fine particles $\left(d_{f}\right)$. They proposed a modification of the equation of Beverloo et al. [2],

$$
Q=C \rho_{m} g^{1 / 2}\left(D-k_{m} \bar{d}_{m}\right)^{5 / 2},
$$

where $\rho_{m}$ is the mixture density, $k_{m}$ is a fitted coefficient different for the two bed microstructures, and $\bar{d}_{m}$ is a mixture diameter which characterizes the empty annulus,

$$
\bar{d}_{m}=X_{f} d_{f}+\left(1-X_{f}\right) d_{c} .
$$

Based on this paper, Humby et al. [7] proposed a semiempirical approach to model the mixture density, taking into account a "flowing density" and found that the empty annulus length is best described using the weighted mean diameter of the mixture [Eq. (3)] but with a small offset. Finally, Chevoir et al. [8] studied experimentally the flow of a binary granular mixture through a sieve, varying the fine mass fraction and the sieve aperture $L$. They proposed an empirical equation for the flow rate of the bidisperse mixture based on the expression of Beverloo et al. [2] for the fine particles,

$$
Q=C \rho g^{1 / 2}\left(L-k d_{f}\right)^{5 / 2}\left[1-\left(\frac{L}{L^{*} d_{c}}\right)^{-n}\right],
$$

where $L^{*}\left(X_{f}\right)$ is the jamming threshold and $n\left(X_{f}\right)$ is a fitted parameter ranging between 3 and 5 .

Despite the broad literature about silo discharge, studies which were dedicated to polydisperse materials appear to be very limited. The current paper is devoted to the experimental investigation of the flow in silo discharges using bidisperse material in cylindrical and rectangular silos. First the experimental setup is described, then we discuss the monodisperse cases and compare our data with the expression of Beverloo et al. [2]. Finally the bidisperse cases are studied, and a model is proposed to predict the flow of a bidisperse mixture. 


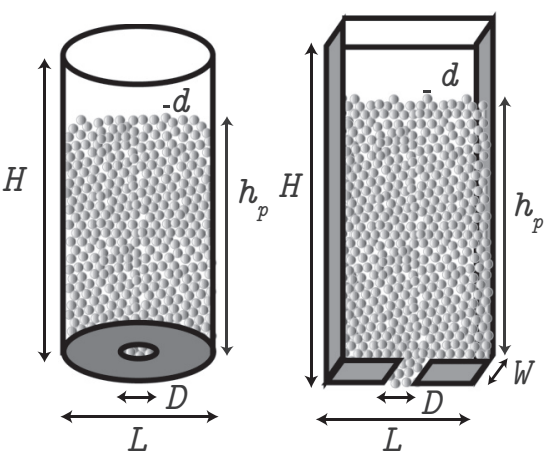

FIG. 1. Schematic experimental apparatus of the cylindrical and the rectangular silos.

\section{EXPERIMENTS}

We use two kinds of flat-bottomed hoppers as shown in Fig. 1. The cylindrical hopper consists of a smooth Perspex cylinder (of diameter $L$ and height $H$ ) with a cylindrical outlet at the center of its bottom whose diameter $D$ was varied. The rectangular hopper was designed to obtain a visualization of the flow. It consists of a smooth and narrow rectangular tube (of height $H$, width $L$, and a thickness $W$ which can be varied). The walls are made of metal, except for the front wall, which is made of Perspex to allow visualization. The apparatus is grounded to prevent the buildup of static electricity. The rectangular outlet located at the center of the bottom spans the thickness $W$ and has a length $D$ which can be varied. The silos' characteristics are summed up in Table I.

The granular material consists of smooth spherical glass beads (density $\rho=2500 \mathrm{~kg} / \mathrm{m}^{3}$, supplied by PottersBallotini) with different diameters reported in Table II. For each outlet aperture, the experiments were first performed using monodisperse beads. With the particles and aperture available, the number of beads in the aperture $(D / d)$ ranges between 1 and 250 . Then different binary mixture combinations were tested corresponding to a size ratio $\left(r=d_{c} / d_{f}\right)$ between the coarse particles $\left(d_{c}\right)$ and the fine particles $\left(d_{f}\right)$ varying from 1.9 to 11.9 (see Table III).

Keeping the outlet closed, the granular column is prepared using the following steps. For a given set of parameters the same total mass $\left(m_{t}\right)$ of granular material was fed into the container from the top, corresponding to a column height of $h_{p} \approx 450 \mathrm{~mm}$ in the rectangular silo and $h_{p} \approx 350 \mathrm{~mm}$ in the cylindrical silo. In the case of binary mixtures, ten layers of the desired fine mass fraction $\left(X_{f}\right)$ previously mixed by hand were poured successively and carefully into the silo. This process is

TABLE I. Silos' characteristics.

\begin{tabular}{lcc}
\hline \hline & Cylindrical & Rectangular \\
\hline$L(\mathrm{~mm})$ & 64 & 60 \\
$H(\mathrm{~mm})$ & 400 & 500 \\
$W(\mathrm{~mm})$ & & $3.5,10$ \\
$D(\mathrm{~mm})$ & 10,20 & Monodisperse: $(1.8-18.5)$ \\
& & Bidisperse: $3.5,5.5,7.5,10,13.5$ \\
\hline \hline
\end{tabular}

TABLE II. Particle mean diameters (the uncertainty is evaluated to be $\approx \pm 10 \%$ ) and symbols used in the figures.

\begin{tabular}{lccccc}
\hline \hline$d(\mu \mathrm{m})$ & Symbol & $d(\mu \mathrm{m})$ & Symbol & $d(\mu \mathrm{m})$ & Symbol \\
\hline 78 & + & 490 & $\bullet$ & 1300 & $\bullet$ \\
114 & $\circ$ & 720 & $\nabla$ & 2090 & $\triangle$ \\
210 & $\boldsymbol{\Delta}$ & 840 & $\boldsymbol{\nabla}$ & 2500 & $\mathbf{\square}$ \\
330 & $\square$ & 1080 & $\times$ & & \\
\hline \hline
\end{tabular}

used to prevent segregation during the filling and to obtain a homogenous state at the scale of the measurement.

After the preparation phase, the column height $\left(h_{p}\right)$ was measured, giving the initial bulk particle volume fraction $\phi_{b}=$ $m_{t} /\left(\rho h_{p} S_{b}\right)$, where $S_{b}$ is the area of the silo cross section $\left(S_{b}=\right.$ $L W$ in the case of the rectangular silo and $S_{b}=\pi L^{2} / 4$ for the cylindrical silo). Then the outlet is quickly opened manually. The grains fall out of the silo and are collected in a vessel; the temporal evolution of the mass $m(t)$ is recorded using an electronic scale (Mettler Toledo 6002S) with a precision of $0.1 \mathrm{~g}$ at $20 \mathrm{~Hz}$. In the rectangular hopper the motion of particles at the front plate is recorded using a video camera (Sony Exmor HDR-XR520) with a resolution of $1920 \times 1080$ pixels at a rate of 25 frames per second. Each experiment is repeated twice to check the reproducibility of the process. For each binary combination, nine mixtures were made from pure fine particles to pure coarse particles with an increment of $X_{f}=12.5 \%$.

A typical temporal evolution of the mass $m(t)$ is shown in Fig. 2(a) (see corresponding movie 1 in the Supplemental Material [9]). The instantaneous mass flow rate $Q_{i}$ is obtained by measuring the local slope of the mass versus time over 20 points (corresponding to $1 \mathrm{~s}$ ). In most cases, the flow is found to be steady, which validates the preparation phase, as can be seen in Fig. 2(b) where the flow rate exhibits a large plateau with small oscillations. The presence of oscillations, which have been previously described by several authors (see, for example, Refs. [10-12]), was not studied in detail. The mean flow rate $Q$ is measured by averaging the instantaneous flow rate over the plateau with the uncertainty given by one standard

TABLE III. Binary combinations with $d_{f}$ as the fine particle diameter, $d_{c}$ as the coarse particle diameter, and $r=d_{c} / d_{f}$ as the size ratio. Symbols used in the figures for the rectangular $(R)$ and cylindrical (C) silos.

\begin{tabular}{lccccc}
\hline \hline Combinations & $d_{f}(\mu \mathrm{m})$ & $d_{c}(\mu \mathrm{m})$ & \multicolumn{1}{c}{$r$} & Symbol R & Symbol C \\
\hline$A$ & 78 & 720 & 9.2 & & + \\
$B$ & 114 & 720 & 6.3 & + & \\
$C$ & 210 & 720 & 3.4 & $\circ$ & $\circ$ \\
$D$ & 330 & 720 & 2.2 & $\boldsymbol{}$ & \\
$E$ & 330 & 1080 & 3.3 & $\square$ & \\
$F$ & 114 & 1300 & 11.4 & $\bullet$ & $\bullet$ \\
$G$ & 210 & 1300 & 5.7 & $\nabla$ & $\nabla$ \\
$H$ & 490 & 1300 & 2.7 & $\bullet$ & $\bullet$ \\
$I$ & 720 & 1300 & 1.8 & $\times$ & $\times$ \\
$J$ & 210 & 2500 & 11.9 & & $\diamond$ \\
$K$ & 720 & 2500 & 3.5 & & $\nabla$ \\
$L$ & 1300 & 2500 & 1.9 & & $\triangle$ \\
\hline \hline
\end{tabular}


(a)

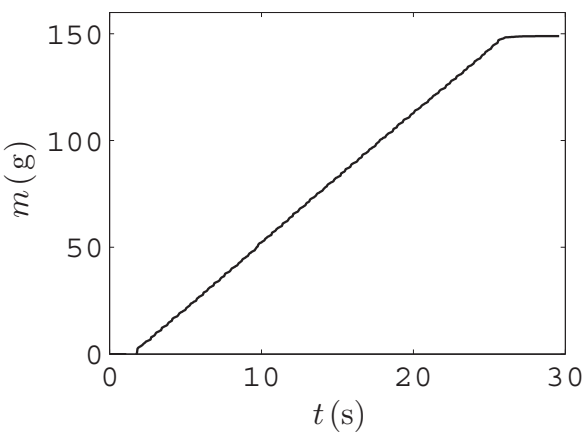

(b)

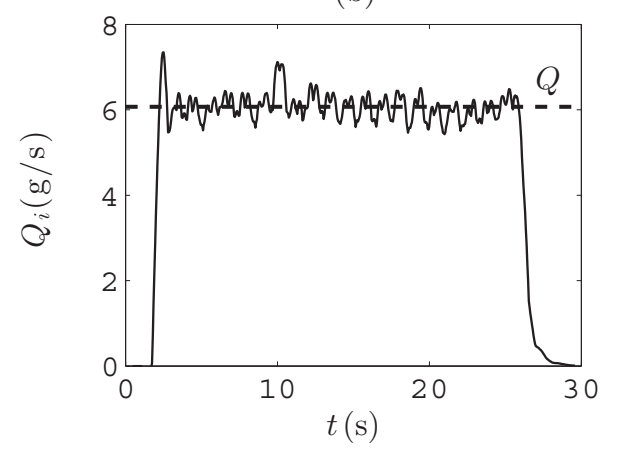

FIG. 2. Temporal evolution for a fine mass fraction $X_{f}=0.625$ of batch $F$ (see Table III) with an outlet diameter of $D=5.4 \mathrm{~mm}$ in the rectangular silo with $W=3.5 \mathrm{~mm}$. (a) Temporal evolution of the mass. (b) Temporal evolution of the instantaneous mass flow rate. The dashed line represents the mean flow rate $Q$.

deviation. The data are available as Supplemental Material in a text file [9] and as Supplemental Material in a MATLAB file [9].

\section{RESULTS AND DISCUSSION}

We will now present the experimental results obtained for the discharge of the cylindrical and of the rectangular silos. We will first focus on the monodisperse flow which will be used as a reference for the bidisperse flow studied in the second part.

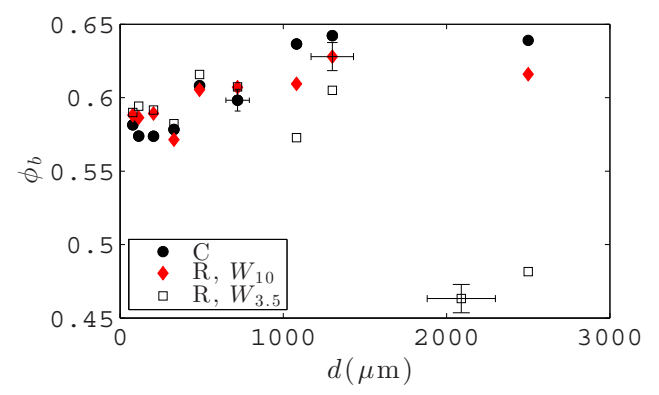

FIG. 3. (Color online) Bulk particle volume fraction versus the particle diameter in the monodisperse case for the cylindrical silo $(\bullet)$ and the rectangular silo with $W=10 \mathrm{~mm}(\checkmark)$ and $W=3.5 \mathrm{~mm}(\square)$. For each symbol only one error bar is shown for the sake of simplicity.
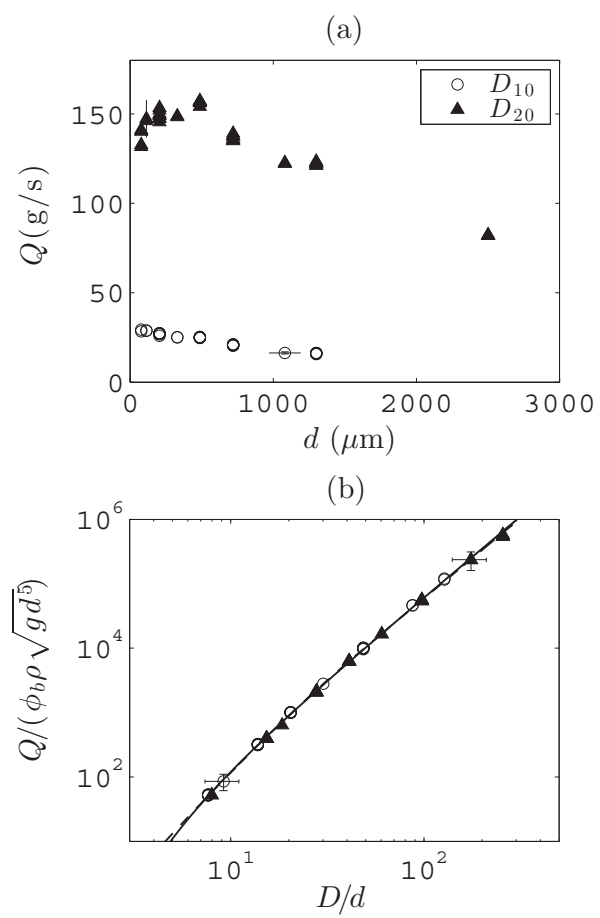

FIG. 4. Flow rate in the monodisperse case for the cylindrical silo for $D=10 \mathrm{~mm}$ (०) and $D=20 \mathrm{~mm}(\mathbf{\Lambda})$ : (a) flow rate $Q$ versus the particle diameter $d$ and (b) flow rate $Q$ made dimensionless by $\phi_{b} \rho \sqrt{g d^{5}}$ versus the outlet diameter $D$ normalized by the particle diameter $d$. The full line represents the expression of Beverloo et al. [2] [Eq. (1)] with $k=1.88$ and $C=0.64$. The dashed line represents the expression of Janda et al. [4] [Eq. (8)] with $\alpha=$ $0.96, \beta=0.09$, and $C^{\prime}=0.75$.

\section{A. Monodisperse flow}

In the monodisperse case, Fig. 3 shows the initial bulk particle volume fraction $\phi_{b}$ as a function of particle diameter for the two apparatuses. The measurements being scattered, $\phi_{b}$ corresponds to the mean value of all the experiments for a given particle diameter with an uncertainty estimated by one standard deviation. We observe that the same tendency is recovered for the cylindrical silo $(\bullet)$ and the largest rectangular silo ( $\bullet$ ). The smallest particles tend to give a slightly looser bed during the filling process than the bigger ones. This effect seems to saturate for the small particles $(d<400 \mu \mathrm{m})$ around 0.58 and for the big particles $(d>1000 \mu \mathrm{m})$ around 0.63 . For the narrowest rectangular silo $(\square)$, the filling process tends to give a loose bed $\phi_{b} \approx 0.58$, whatever the particle diameter, except for the biggest particle where the particle volume fraction drops to $\phi_{b} \approx 0.47$ due to the fact that there is only one layer of particle in the thickness of the silo.

We now turn to the study of the stationary flow of the monodisperse cases. For the cylindrical silo, Fig. 4(a) shows the flow rate $Q$ versus the particle diameter $d$ for the two outlet diameters $D=10 \mathrm{~mm}$ (०) and $D=20 \mathrm{~mm}(\boldsymbol{\Lambda})$. The flow rate exhibits mainly a decrease when the particle diameter increases and a strong dependence with the outlet diameter. To test the expression of Beverloo et al. [2] [Eq. (1)], the flow rate, made dimensionless by $\phi_{b} \rho \sqrt{g d^{5}}$, is plotted in Fig. 4(b) versus the outlet diameter $D$ normalized by the particle diameter $d$. 


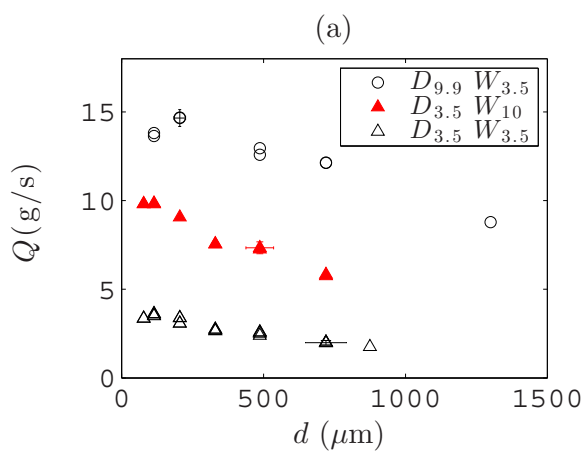

(b)

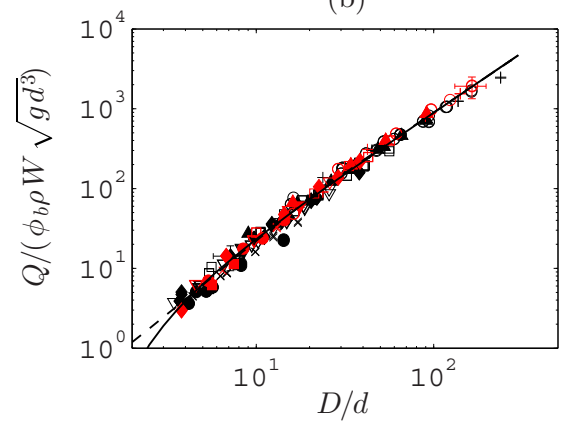

FIG. 5. (Color online) Flow rate in the monodisperse case for the rectangular silo. (a) Flow rate $Q$ versus the particles diameter $d$ for $W=3.5 \mathrm{~mm}$ with $D=3.5 \mathrm{~mm}(\triangle)$ and $D=9.9 \mathrm{~mm}$ (०) and for $W=10 \mathrm{~mm}$ with $D=3.3 \mathrm{~mm}(\mathbf{\Delta})$. (b) Flow rate $Q$ made dimensionless by $\phi_{b} \rho W \sqrt{g d^{3}}$ versus the outlet length $D$ made dimensionless by the particle diameter $d$ for the different particle diameters (symbol given in Table II) and for the two thicknesses $W=$ $3.5 \mathrm{~mm}$ (black symbols) and $W=10 \mathrm{~mm}$ [red (gray) symbols]. The full line represents the expression of Beverloo et al. [2] [Eq. (5)] with $k=1.36$ and $C=0.91$. The dashed line represents the expression of Janda et al. [4] [Eq. (8)] with $\alpha=0.66, \beta=0.11$, and $C^{\prime}=0.90$.

The data are all superimposed, suggesting that $D / d$ is the good control parameter, and well represented by the expression of Beverloo et al. [2] (see the dashed line in the figure) with the fitting parameters $k=1.88$ and $C=0.64$ (obtained using the least squares method), which closely match those reported in previous papers $[1,13]$.

In the case of the rectangular silo, the orifice being a slit, i.e., spanning with the thickness, we first have tested the role played by the thickness and the length of the outlet. Figure 5(a) shows the flow rate $Q$ versus the particle diameter $d$ in the rectangular silo for the two thicknesses $[W=10 \mathrm{~mm}$ : gray symbols (red) and $W=3.5 \mathrm{~mm}$ : black symbol] and for two outlet lengths $(D \approx 3.5 \mathrm{~mm}$ : triangles and $D \approx 10 \mathrm{~mm}$ : circles). Again, the flow rate decreases slightly when increasing the particles diameter as described by Beverloo et al. [2]. In the graph, the two upper curves correspond to runs with the same outlet surface $S_{o}=W D$ but with two different thicknesses and lengths $[W=3.5$ and $D=9.9 \mathrm{~mm}$ (०) and $W=10$ and $D=3.3 \mathrm{~mm}(\Delta)]$. It is clear that the two dimensions do not play the same role, the flow being faster for the largest length $D$. Taking as a reference the lowest curve corresponding to the square outlet ( $D \approx 3.5$ and $W \approx 3.5 \mathrm{~mm}: \Delta$ ), the comparison with the two previous curves suggests that the flow rate is proportional to $W$ and has a stronger variation with $D$.

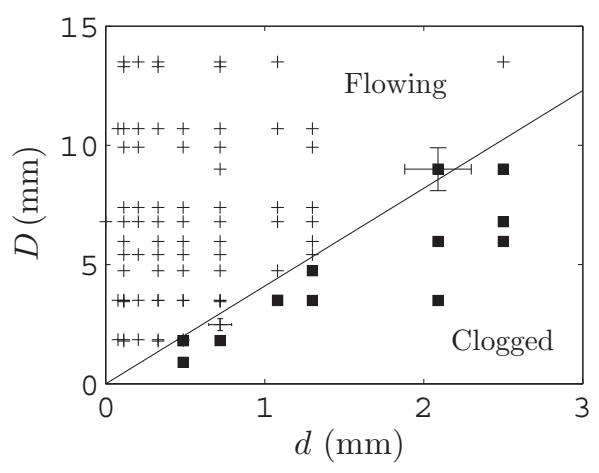

FIG. 6. Phase diagram of the two regimes observed (flowing: + and clogged: $\square)$ in the $(D, d)$ plane for the rectangular silo with $W=3.5 \mathrm{~mm}$. The solid line corresponds to $D=4.1 d$.

This behavior can be understood with reference to the concept of the free fall arch. Due to the outlet configuration, it is probable that no arch can develop over the thickness of the silo, so we expect that the free fall arch scales with the length $D$. With these observations, we can adapt the expression of Beverloo et al. [2] [Eq. (1)] and write it for the rectangular silo,

$$
Q=C \rho \phi_{b} g^{1 / 2} W(D-k d)^{3 / 2} .
$$

Figure 5(b) shows the flow rate $Q$, made dimensionless by $\phi_{b} \rho W \sqrt{g d^{3}}$, versus the outlet length $D$ normalized by the particle diameter $d$ for the different particle diameters (symbol given in Table II) and the two available thicknesses. This choice of dimensionless parameter results in a single curve for all of the data, which suggests that the scaling on the thickness is appropriate. The data are well represented by the expression of Beverloo et al. [2] [Eq. (5), see the full line in the figure] with the adjusted parameters $k=1.36$ and $C=0.91$ (using the least squares method). These parameters are in good agreement with those reported in previous papers and are slightly different from the cylindrical ones $[1,4,14]$.

Finally, we have studied the limit of jamming in the narrowest rectangular silo $(W=3.5 \mathrm{~mm}$ ) varying the length of the outlet $D$ and the particle diameters $d$. In Fig. 6, the two regimes observed (flowing: + and clogged: $\mathbf{\square})$ are represented in the $(D, d)$ plane. The jamming seems to occurs for $D<4.1 d$, which is compatible with previous results in three dimensional $[15,16]$ and two dimensional $[17,18]$ configurations.

The study of the flow of monodisperse particles in our apparatus has shown that our data are well described by the expression empirically developed first by Beverloo et al. [2]. The monodisperse study will be used in the following as a reference for the bidisperse flow. The dependence on the thickness of the silo being well described by a linear variation, in the rest of this study the results are obtained with the narrowest silo corresponding to the thickness $W=3.5 \mathrm{~mm}$.

\section{B. Bidisperse flow}

In the case of a bidisperse mixture, the process of filling the silo is described in Sec. II. The bulk particle volume fraction obtained is given in Fig. 7 as a function of the fine mass fraction 


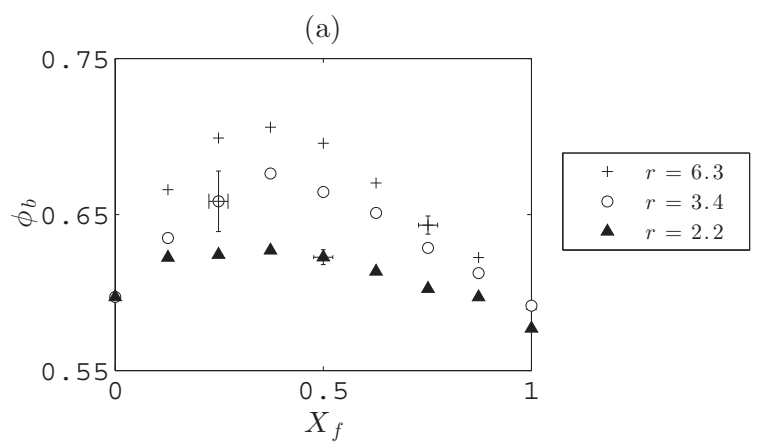

(b)

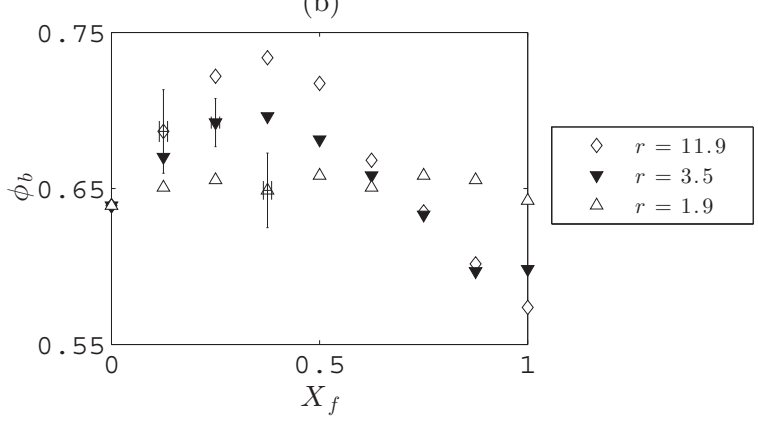

FIG. 7. Bulk particle volume fraction versus the fine mass fraction $\left(X_{f}\right)$ (a) for the rectangular silo with $d_{c}=720 \mu \mathrm{m}$ and various $d_{f}=$ $114 \mu \mathrm{m}(+), 210 \mu \mathrm{m}(\mathrm{o})$, and $330 \mu \mathrm{m}(\boldsymbol{\Delta})$ and (b) for the cylindrical silo with $d_{c}=2500 \mu \mathrm{m}$ and various $d_{f}=210 \mu \mathrm{m}(\diamond), 720 \mu \mathrm{m}(\mathbf{\nabla})$, and $1300 \mu \mathrm{m}(\triangle)$ (see the corresponding size ratios $r$ in the legend).

for both silo geometries. As shown in the literature [6], $\phi_{b}$ depends on the fine mass fraction $\left(X_{f}\right)$ and on the size ratio $(r)$. For low size ratios, $\phi_{b}$ is nearly constant, whereas for high size ratios, $\phi_{b}$ goes through a maximum, around $X_{f} \approx 0.375$, corresponding to dense packing where the fine particles fill the holes between the coarse particles.

In some cases, segregation occurs during silo discharge. This can be seen, for example, in Fig. 8(a) where the flow rate is plotted versus time for different fine mass fractions $\left(X_{f}=0,0.25,1\right)$ for a mixture with a size ratio of $r=11.4$

(b)
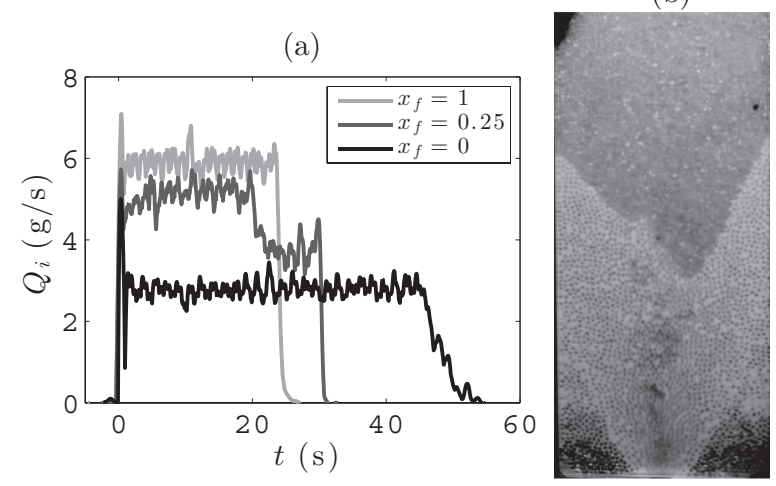

FIG. 8. (a) Flow rate versus time for different fine mass fractions $\left(X_{f}=0,0.25,1\right)$ from black to light gray for the rectangular silo with $r=11.4$ (batch $F$ in Table III) and $D=5.5 \mathrm{~mm}$. (b) Image of the corresponding movie for $X_{f}=0.25$ after $17.5 \mathrm{~s}$ of flow (the width of the image corresponds to the width of the silo $L=60 \mathrm{~mm}$ ).

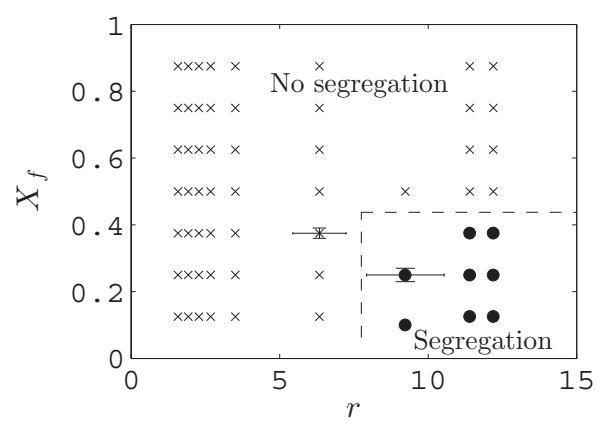

FIG. 9. Phase diagram of the two regimes of segregation observed ( $\mathrm{x}$ : no segregation and $\bullet$ : segregation) in the $\left(X_{f}, r\right)$ plane for the cylindrical silo with $D=20 \mathrm{~mm}$. The dashed lines are indicative of the transition.

(batch $F$ in Table III) in the rectangular silo with an outlet size of $D=5.5 \mathrm{~mm}$. The pure coarse $\left(X_{f}=0\right)$ and fine particles $\left(X_{f}=1\right)$ exhibit a constant flow rate with time, whereas in the case of a fine fraction of $X_{f}=0.25$, the flow rate is first constant and then decreases towards the value corresponding to the pure coarse particles after $20 \mathrm{~s}$. In Fig. 8(b) an image of the particles is shown for $X_{f}=0.25$, after $17.5 \mathrm{~s}$ of flow (see the Supplemental Material movie 2 [9]). It can be clearly seen that the upper part of the silo is filled mainly with the coarse particles and not with the initial mixture as in the lower part. This means that a segregation phenomenon has occurred during the discharge, which can explain the behavior of the corresponding flow rate in Fig. 8(a): Initially we observe the flow rate of the mixture, then that of the segregated coarse particles. From the flow rate variation with time we can extract the cases where some segregation occurs. This is performed in Fig. 9(a) in the $\left(X_{f}, r\right)$ plane for the cylindrical silo with $D=20 \mathrm{~mm}$. In this silo geometry, segregation occurs for large size ratios and low fine mass fractions $\left(r \geq 9.2\right.$ and $X_{f} \leq$ $0.375)$. The same behavior is recovered in the rectangular silo where segregation is observed for $r \geq 6$ and $X_{f} \leq 0.375$. As shown by Samadani et al. [19] segregation occurs only during avalanches at the free granular surface and not in the bulk flow. In our silo's geometry, the segregation process was not studied in detail, but we observe that the segregation tends to develop only at the end of the flow. In the rest of this study, we will focus on the flow rate of the mixture. In the few cases where segregation occurs, we will use only the early time period of the experiments, before the development of segregation.

Figures 10(a) and 10(b) show the mean flow rate of the mixture versus the fine mass fraction for a given batch and various outlet sizes in the rectangular and the cylindrical silos. We find that the flow rate of the mixture always exceeds the rate predicted by a simple mixture law that uses the flow rates of the purely coarse particles and the purely fine particles. We observe two behaviors: First the flow rate increases with the fine mass fraction and, then, it tends to stabilize or slightly decrease.

To understand this behavior, we have developed a model based on the recent experimental paper of Janda et al. [4]. They proposed an expression for the flow rate of particles through orifices for the velocity and density profiles at the exit as a function of the horizontal position $x$ in a two 

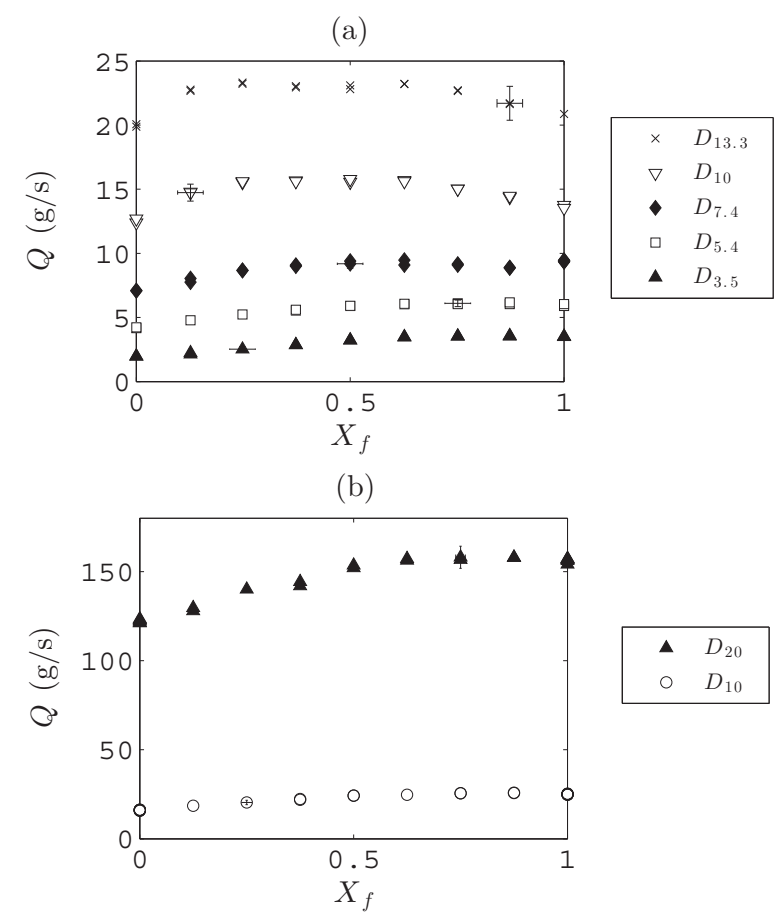

FIG. 10. Mean flow rate $(Q)$ of the mixture versus the fine mass fraction $\left(X_{f}\right)$ for various outlet sizes $D$ (see the legend) for (a) the rectangular silo with $r=6.3$ (batch $B$, see Table III) and (b) the cylindrical silo with $r=2.7$ (batch $H$ ).

dimensional silo. They found that the velocity profile is selfsimilar, whatever the diameter of the aperture $D$, and wrote $v(x)=v_{o} \sqrt{1-(2 x / D)^{2}}$. They obtained that the velocity $v_{o}$ at the center of the outlet corresponds to a free fall from a hypothetical arch, scaling with $D$, as commonly accepted

$$
v_{o}=\sqrt{\gamma g D} .
$$

Their equation suggests that for a given outlet diameter, the particle velocities at the outlet are the same whatever the particle diameters. In the same way, the density profile is found to be self-similar with $\phi(x)=\phi_{o}\left[1-(2 x / D)^{2}\right]^{0.22}$ where the variation in density at the center of the outlet $\phi_{o}=\phi_{\infty}\left[1-\alpha_{1} e^{-D / 2 \alpha_{2}}\right]$ corresponds to an asymptotic growth with the outlet size which can be fitted by an exponential saturation. They concluded that, for small aperture, a dilatancy occurs in order to avoid the formations of arches in order to maintain the material flowing. Then they infer the concept of the empty annulus is not necessary.

To adapt this concept to our configurations, we suppose that the asymptotic value of the volume fraction for big orifices is proportional to the initial bulk volume fraction $\left(\phi_{\infty}=\xi \phi_{b}\right)$. We assume that the fitting parameter $\alpha_{2}$ is proportional to the particle diameter $d$ as suggested by the authors. The particle volume fraction at the center of the outlet is then given by

$$
\phi_{o}=\xi \phi_{b}\left[1-\alpha e^{-\beta(D / d)}\right]=\xi \phi_{b} \Gamma_{d},
$$

where we note that $\Gamma_{d}$ is the geometrical factor which depends on the particle size (within the number of beads in the aperture $D / d)$ and which characterizes the dilatancy at the outlet. The flow rate being defined by $Q=\rho \iint_{S_{o}} \phi v d S=C_{1} \rho \phi_{o} v_{o} S_{o}$, we can finally adapt the relation of Janda et al. [4] to our configurations,

$$
Q=C^{\prime} \rho \phi_{b} \Gamma_{d} \sqrt{g D} S_{o} .
$$

This expression represents well our data in the monodisperse cases: See the dashed lines in Figs. 4(b) and 5(b) with the fitting parameters, obtained using the least squares method $C^{\prime}=0.75, \alpha=0.96$, and $\beta=0.09$ in the cylindrical silo and $C^{\prime}=0.90, \alpha=0.66$, and $\beta=0.11$ in the rectangular silo. The fitting parameters are in good agreement compared to those of Janda et al. [4]. In the range of explored parameters, we are not able to distinguish the expression of Beverloo et al. [2] from the expression of Janda et al. [4].

In the case of bidisperse flow, we follow Janda et al. [4], who concluded that the self-similarity of the profiles indicates the generality of the mechanism that controls the flow rate. Then we expect that the velocity profile of the mixture keeps its self-similar form, which is independent of the particle diameter [Eq. (6)].

Concerning the volume fraction, by definition we can decompose it into $\phi(x)=\phi_{f}(x)+\phi_{c}(x)$, where $\phi_{f}=X_{f} \phi$ is the fine volume fraction and $\phi_{c}=\left(1-X_{f}\right) \phi$ is the coarse volume fraction. This remains valid at the center of the outlet where

$$
\phi_{o}=\phi_{o f}+\phi_{o c} .
$$

We first assume that for each particle size, the dilatancy expression [Eq. (7)] is still valid independent of the other particle size,

$$
\phi_{o}=\xi \phi_{b f} \Gamma_{d_{f}}+\xi \phi_{b c} \Gamma_{d_{c}},
$$

where $\phi_{b f}=X_{f} \phi_{b}$ and $\phi_{b c}=\left(1-X_{f}\right) \phi_{b}$, respectively, are the fine and coarse initial bulk volume fractions.

Second we assume that the self-similarity of the density profile remains valid for the mixture, leading by integration to the flow rate of a bidisperse mixture,

$$
Q=C^{\prime}\left[X_{f} \Gamma_{d_{f}}+\left(1-X_{f}\right) \Gamma_{d_{c}}\right] \rho \phi_{b} \sqrt{g D} S_{o} .
$$

This relation implies a complex variation in the flow rate $Q$ with the fine mass fraction $X_{f}$ as the bulk particle volume fraction $\phi_{b}$ also depends on $X_{f}$ (Fig. 7). This is compatible with the observations made in Figs. 10(a) and 10(b).

To simply test this model, we introduce the bulk velocity $v_{b}=Q /\left(\rho \phi_{b} S_{b}\right)$, where $S_{b}$ is the area of the silo cross section, which should vary linearly with the fine mass fraction,

$$
v_{b}=X_{f} v_{b f}+\left(1-X_{f}\right) v_{b c}
$$

where $v_{b i}=C^{\prime} \Gamma_{d_{i}} \sqrt{g D} S_{o} / S_{b}$ represents the bulk velocity in the pure case $(i=f, c)$. In Fig. 11 this velocity is plotted versus the fine mass fraction for the same data as in Fig. 10. It is very interesting to note that, as predicted by Eq. (12), the data for a given batch and a given outlet diameter exhibit a linear trend. The dashed lines correspond to the best linear fit excluding the pure cases $X_{f}=0$ and $X_{f}=1$. To further test the validity of Eq. (12), we extract from the fitting parameter the value of the bulk velocity for the pure case $v_{b i}$. In Fig. 12 we plot these extrapolated velocities, made dimensionless by $\sqrt{g d_{i}^{5}} / S_{b}$ in the cylindrical case and by $W \sqrt{g d_{i}^{3}} / S_{b}$ in the rectangular case, versus $D / d$ and compare them to the adjusted expressions 


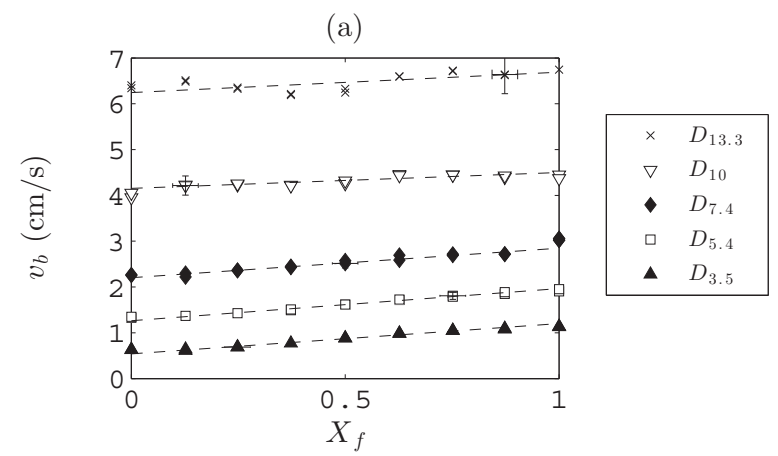

(b)

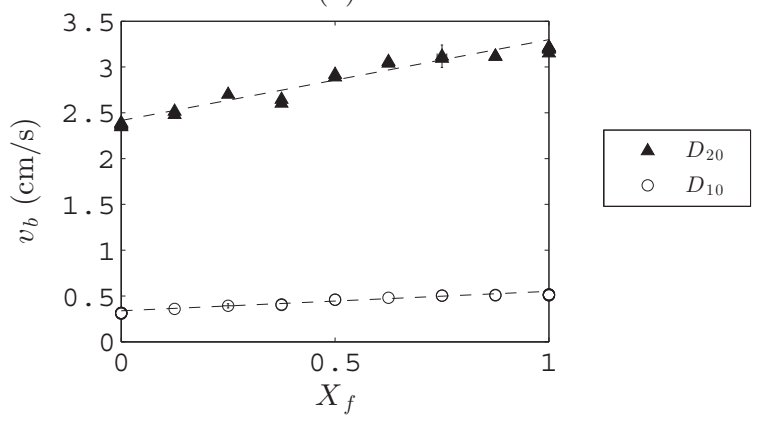

FIG. 11. Bulk velocity $\left(v_{b}\right)$ versus the fine mass fraction $\left(X_{f}\right)$ for the same data as in Figs. 10(a) and 10(b). The dashed lines represent the best linear fit excluding the pure cases $\left(X_{f}=0\right.$ and $\left.X_{f}=1\right)$ for each outlet length $D$.

(a)

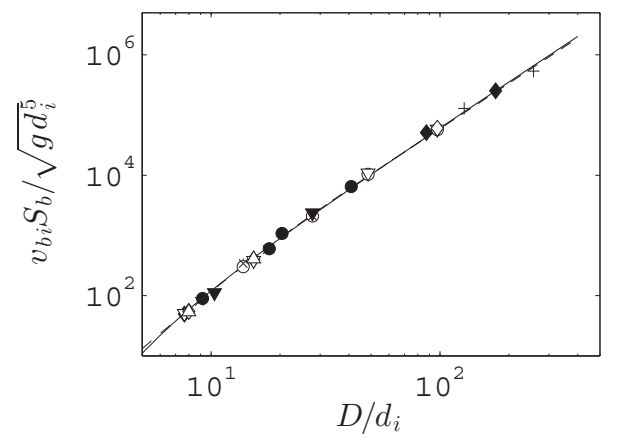

(b)

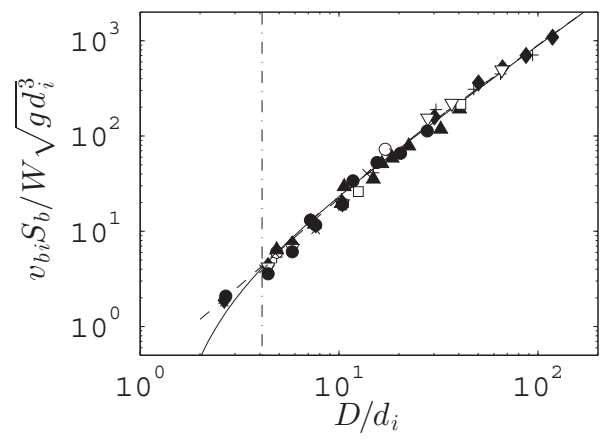

FIG. 12. Nondimensional bulk velocity of the pure case $v_{b i}(i=$ $f, c$ ) versus the outlet length $D$ made dimensionless by the particle diameter $d_{i}$ for all batches (symbol given in Table III) for (a) the cylindrical silo and (b) the rectangular silo. The vertical dashed-dotted line represents the limit of jamming for the monodisperse case. The full and dashed lines are the same as in Figs. 4(b) and 5(b).

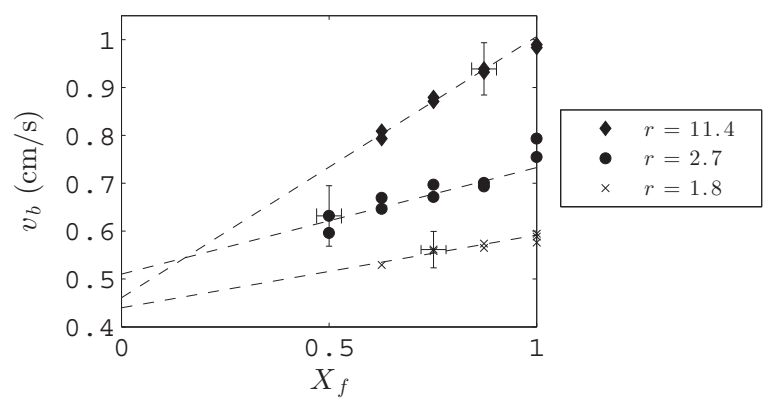

FIG. 13. Bulk velocity $\left(v_{b}\right)$ versus the fine mass fraction $\left(X_{f}\right)$ for the rectangular silo with $D=3.5 \mathrm{~mm}, d_{c}=1300 \mu \mathrm{m}$, and various $d_{f}=114 \mu \mathrm{m}(\diamond) ; 490 \mu \mathrm{m}(\bullet)$, and $720 \mu \mathrm{m}(\times)$ (see the corresponding size ratios $r$ in the legend). The dashed lines represent the best linear fit excluding the pure case $\left(X_{f}=1\right)$ for each batch.

obtained in the case of monodisperse experiments [Figs. 4(b) and 5(b)]. We observe good agreement with the data, which gives confidence in the use of a simple mixture law on the bulk velocity to obtain the flow rate of the mixture [Eq. (12)]. This seems to show that there is not a different behavior between the coarse continuous phase and the fine continuous phase as previously suggested [6,7]. Moreover this model also suggests that a mixture diameter cannot be simply defined to adapt the monodisperse expressions to predict the bidisperse flow rate as often proposed in the literature.

Finally, it is interesting to note that in Fig. 12(b) we obtained data below the limit of jamming (vertical dashed-dotted line in the graph) where no direct measurement is possible. These new data are still in good agreement with the adjusted expressions of Beverloo et al. [2] and Janda et al. [4]. They have been obtained from Fig. 13 where we have plotted the bulk velocity $v_{b}$ versus the fine mass fraction $X_{f}$ for the rectangular silo with $D=3.5 \mathrm{~mm}$ and different batches with the same coarse diameter $d_{c}=1300 \mu \mathrm{m}$. The coarse particles are below the limit of jamming for this outlet diameter, but we observe that they are able to flow when they are mixed with smaller particles until a mass fraction of about $50 \%$. This remains true even for a small size ratio as seen in the data where $r=1.8\left(d_{f}=720 \mu \mathrm{m}: \times\right)$. The dashed lines represent the best linear fit for each batch, excluding the pure fine case $\left(X_{f}=1\right)$. They converge at $X_{f}=0$ giving the extrapolated bulk velocity which would correspond to particles of diameter $d=1300 \mu \mathrm{m}$ flowing in an outlet of length $D=3.5 \mathrm{~mm}$, i.e., less than three beads in the aperture. From a numerical and experimental study in a cylindrical silo, Pournin et al. [15] suggested that the jamming of bidisperse mixture follows the same expression than that of monodisperse assemblies if we consider the volume-averaged diameter of the constituting bed [Eq. (3)]. This relation does not seem to represent our results, however a more complete experimental study on this point deserves to be performed.

\section{CONCLUSION}

We have experimentally studied the flow discharge in a cylindrical and a rectangular silo using both monodisperse and bidisperse mixtures of spherical glass beads. We have shown that a stationary regime is obtained during the discharge. In the 
case of monodisperse particles, the flow rate is well adjusted both by the expression of Beverloo et al. [2] [Eq. (1)] and the expression of Janda et al. [4] [Eq. (8)]. In the case of a rectangular silo, the expression of Beverloo et al. [2] has been successfully modified to take into account the effect of the difference between the thickness and the length of the outlet, the orifice being a slit [see Eq. (5)].

We have developed a simple model based on the experiment of Janda et al. on monodisperse particles [4] to predict the flow rate of the bidisperse granular media. This model supposes that the particle velocities at the outlet are the same whatever the particle diameters. Then it assumes that the dilatancy expression [Eq. (7)] is still valid for each particle size, independent of the other particle size. Finally it presumes that the self-similarity of the density profile remains valid for the mixture. Based on all these hypotheses, we propose an expression for the flow rate of a bidisperse mixture [Eq. (11)]. This model predicts that the bulk velocity $v_{b}=Q /\left(\rho \phi_{b} S_{b}\right)$ follows a simple mixture law [Eq. (12)] which was validated experimentally. Furthermore, our model suggests that a mixture diameter cannot be simply defined to adapt the monodisperse expressions to predict the bidisperse flow rate as commonly seen in the literature.

Finally we have shown that coarse particles can be transported in small outlets, below the jamming conditions up to a mass fraction of about $50 \%$ and even for a small size ratio (1.8 in this study). The extrapolated bulk velocities of the pure coarse particles below the limit of jamming are still in good agreement with the expressions of Beverloo et al. [2] and Janda et al. [4].

The new data obtained could be used as a benchmark to test theoretical models. In particular, the development of a continuous rheology [20] has been found to successfully predict the flow of particles in many geometries [21]. In the case of the discharge of a granular silo, interesting results have been obtained using this rheology in numerical studies [2224], however the dependence of the flow rate with the particle diameters remains an open question in a continuous model. Concerning bidisperse flow, the simple mixture law obtained for the bulk velocity suggests that the mixture can potentially be seen as a continuous media. It would be interesting now to use our data to test a mixture rheology [25,26] in a continuous model as previously performed for silo discharge of monodisperse particles or flow down an inclined plane for polydisperse particles [26].

\section{ACKNOWLEDGMENTS}

We would like to thank P. Cervetti, S. Noel, and F. Ratouchniak for technical assistance, F. Marconi and K. Toujri for experimental work, and J. E. Butler for critical reading. Université de Béchar and Laboratoire IUSTI are gratefully acknowledged by M.B.
[1] R. M. Nedderman, U. Tuzün, S. B. Savage, and G. T. Houlsby, Chem. Eng. Sci. 11, 1597 (1982).

[2] W. A. Beverloo, H. A. Leniger, and J. V. de Velde, Chem. Eng. Sci. 15, 260 (1961).

[3] B. Tighe and M. Sperl, Granular Matter 9, 141 (2007).

[4] A. Janda, I. Zuriguel, and D. Maza, Phys. Rev. Lett. 108, 248001 (2012).

[5] R. L. Brown and J. C. Richards, Trans. Inst. Chem. Eng. 38, 243 (1960).

[6] P. Arteaga and U. Tüzün, Chem. Eng. Sci. 45, 205 (1990).

[7] S. Humby, U. Tüzün, and A. B. Yu, Chem. Eng. Sci. 53, 483 (1998).

[8] F. Chevoir, P. Gaulard, and N. Roussel, Eur. Phys. Lett. 79, 14001 (2007).

[9] See Supplemental Material at http://link.aps.org/supplemental/ 10.1103/PhysRevE.90.032201 for a movie of the silo discharge experiment corresponding to the results shown in Fig. 2, the data in a text file, the data in a MATLAB file, and a movie of the silo discharge experiment corresponding to the results shown in Fig. 8(a) for $X_{f}=0.25$.

[10] X.-I. Wu, K. J. Måløy, A. Hansen, M. Ammi, and D. Bideau, Phys. Rev. Lett. 71, 1363 (1993).

[11] T. Le Pennec, K. J. Måløy, A. Hansen, M. Ammi, D. Bideau, and X.-I. Wu, Phys. Rev. E 53, 2257 (1996).

[12] R. O. Uñac, A. M. Vidales, O. A. Benegas, and I. Ippolito, Powder Technol. 225, 214 (2012).
[13] J. E. Hilton and P. W. Cleary, Phys. Rev. E 84, 011307 (2011).

[14] C. Mankoc, A. Janda, A. Arévalo, J. M. Pastor, I. Zuriguel, A. Garcimartín, and D. Maza, Granular Matter 9, 407 (2007).

[15] L. Pournin, M. Ramaioli, P. Folly, and T. M. Liebling, Eur. Phys. J. E 23, 229 (2007).

[16] A. Janda, D. Maza, A. Garcimartin, E. Kolb, J. Lanuza, and E. Clément, Eur. Phys. Lett. 87, 24002 (2009).

[17] K. To, P.-Y. Lai, and H. K. Pak, Phys. Rev. Lett. 86, 71 (2001).

[18] A. Janda, R. Harich, I. Zuriguel, D. Maza, P. Cixous, and A. Garcimartín, Phys. Rev. E 79, 031302 (2009).

[19] A. Samadani, A. Pradhan, and A. Kudrolli, Phys. Rev. E 60, 7203 (1999).

[20] P. Jop, Y. Forterre, and O. Pouliquen, Nature (London) 441, 727 (2006).

[21] Y. Forterre and O. Pouliquen, Annu. Rev. Fluid Mech. 40, 1 (2008).

[22] K. Kamrin, Int. J. Plast. 26, 167 (2010).

[23] L. Staron, P.-Y. Lagrée, and S. Popinet, Phys. Fluids 24, 103301 (2012).

[24] J. Chauchat and M. Médale, J. Comput. Phys. 256, 696 (2014).

[25] P. G. Rognon, J.-N. Roux, M. Naaïm, and F. Chevoir, Phys. Fluids 19, 058101 (2007).

[26] B. Marks, P. Rognon, and I. Einav, J. Fluid Mech. 690, 499 (2012). 